\title{
Model Identification and Control for Nonlinear Discrete-time System with Time Delay: A Support Vector Machine Approach
}

\author{
Weimin Zhong Hongwei Ge Feng Qian \\ State-Key Laboratory of Chemical Engineering, Automation Institute of East China University of Science and \\ Technology, Shanghai 200237, P.R. China
}

\begin{abstract}
In this paper, a design procedure of support vector machine (SVM) based model identification and control strategy for stable nonlinear discrete-time process with input-output form is proposed. In order to implement the control structure, the both inverse and straight model representation and identification methods are addressed in detail. The control of a simulated continuous-stirred tank reactor (CSTR) process illustrates the proposed design procedure and the properties of the SVM based model identification, direct inverse model control and internal model control for nonlinear systems.
\end{abstract}

Keywords: Support vector machine, Internal model control, Nonlinear system, Inverse model, Identification

\section{Introduction}

In nonlinear control literature, several techniques have been developed to control nonlinear plants subject to uncertainties and disturbances: among them, nonlinear internal model control (NIMC) method has played a significant role due to its properties in good robustness against disturbances and model mismatch [1]-[2]. The basic idea of nonlinear internal model control is illustrated in Fig.1. It consists of the controller and a predictive model of the process, i.e., the internal model .The internal model loop uses the difference between the outputs of the process and of the internal model, which represents the effect of disturbances and model mismatch. In the past years, IMC has been extensively studied in the case of a linear modeling of the process with good robustness properties against disturbance and mode mismatch. But in the most applications of industrial process control such as chemical and power plant, it is difficult in modeling such complex and nonlinear plant using traditional methods. Developments of NIMC have been proposed for continuous-time systems [3]-[4] and for discretetime systems [5] using neural network and fuzzy theory. Generally, these methods have at least one of the following restrictive conditions: (1) the sample time should be long enough to allow an iterative search for the controller output; (2) the generalization ability is poor when using neural network because neural network is constructed on experience risk minimization principle; (3) the objective maybe has a local minimum and training speed is slow in the case of using neural network; (4) modeling needs more prior knowledge and relies much more on experts in the case of using fuzzy theory. This paper develops a SVM based NIMC method which is free of all these limitations.

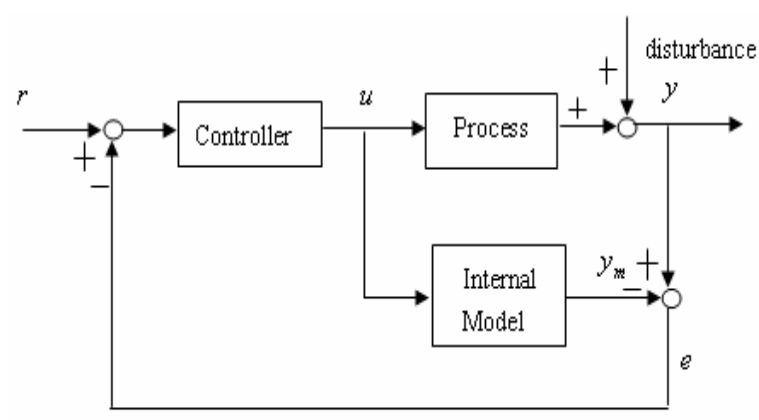

Fig.1: Internal model control system.

Recently a new kind of learning machine called support vector machine (SVM) [6]-[7] was presented and has been used for classification, function regression, and time series prediction, etc [6]-[8]. SVM, with simple topological structure and good generalization capability, is a great achievement in machine learning area in recent years. Its basic idea is to map input data into a high dimensional feature space via a nonlinear mapping technique (kernel dot product trick), and to carry out linear classification or regression in the feature space. SVM solutions who are global optimal and unique are characterized by convex quadratic optimization problems, which are 
typically solved in dual space. SVM has excellent capability in generalization because it is constructed on structure risk minimization principle. Because SVM can approximate nonlinear functions with arbitrary precision [6], now some researcher has studied the application of SVM in nonlinear system identification and control [9]-[10].

In [11], a simple SVM internal model with RBF kernel function was built to control the greenhouse environment problem. And in our earlier work [12], a SVM based inverse model for nonlinear system identification and control was studied. All these are initial work. In this paper, we detail the SVM based model identification and internal model control for nonlinear system. Like neural network, the first stage for the SVM based identification and control of an unknown dynamic system is the development of an accurate model of the plant under consideration. Obtaining such a model involves a training phase where the SVM is presented with a set of previously collected input-output data of the system. In this case, the training phase can be accomplished only off-line by connecting the SVM model in an open-loop configuration with the unknown plant which is excited with a set of selected inputs in order to measure the corresponding outputs. Unlike neural network's gradient based iterative method under experience risk minimization principle, SVM uses kernel dot product trick and convex quadratic optimization abiding by structure risk minimization principle, which guarantees that the solutions can be obtained by oncethrough operation and are global optima. So if the inversion of unknown dynamic system exists, the SVM can obtain the inverse model and straight model of the system easily, quickly and accurate using the same set of input-output data. Though standard SVM can only build off-line model (non-adaptive training), it performs much more outstandingly at dealing with nonlinear time-invariable system than neural network does.

We propose here a general design procedure of SVM internal model identification and control systems. We first introduce the SVM identification of internal model and inverse model. The time delay will be taken into account, and we will find that the time delay dose not increase the difficulty of modeling, which is of importance for the identification. Then, we study the SVM based control including the direct inverse control and internal model control. And last, we will use a CSTR example to illustrate the SVM based identification and control performance. The advantages of SVM are exploited through the paper, in particular, the ability of SVM for nonlinear blackbox inverse and straight modeling.

\section{SVM based nonlinear modeling}

In this paper, a stable single input-single output process is investigated. We consider the process to be described by the following discrete-time deterministic nonlinear input-output model [2]:

$$
\left\{\begin{array}{c}
x_{p}(k+1)=h\left(x _ { p } \left\{\begin{array}{l}
k \\
k-n+1
\end{array}, u\left\{\begin{array}{l}
k-d \\
k-d-m+1
\end{array}\right)\right.\right. \\
y_{p}(k+1)=x_{p}(k+1)+p(k+1)
\end{array}\right.
$$

where $\quad x_{p}\left\{\begin{array}{l}k-n+1 \\ k\end{array}\right.$ denotes the known state $\left\{x_{p}(k), \cdots, x_{p}(k-n+1)\right\}$ and $u\left\{\begin{array}{l}k-d \\ k-d-m+1\end{array}\right.$ denotes the set of $m$ past control inputs $\{u(k-d), \cdots, u(k-d-m+1)\} ; d \in[0,1,2, \cdots]$ is the time delay; $h$ is an unknown nonlinear function; $y_{p}$ is the output, the effect of disturbance being modeled by $p$. Here, provided that $p=0$, and in the simulation, the effect of disturbance will be considered.

Efficient black-box modeling needs the training sequences are of sufficient size and distribution. Under the assumption that the system is zero initial, we use a random series of $u_{t}$ (size is $N_{t}$, i.e., $u_{t}(1), \ldots u_{t}\left(N_{t}\right)$ ) full of the input domain to generate the series of $y_{p t}\left(y_{p t}(1), \cdots, y_{p t}\left(N_{t}\right), y_{p t}\left(N_{t}+1\right), \cdots, y_{p t}\left(N_{t}+d\right), \cdots\right)$ . Note that, we measure the output at the same time when $u_{t}$ is added to the system, and because the system has time delay and is auto regression, $y_{p t}$ has a longer series. And the SVM based true model at sample instant time $k$ is of the form:

$$
\begin{gathered}
y_{p}(k+1)=\phi\left(y _ { p t } \left\{\begin{array}{l}
i \\
i-n+1
\end{array}, u_{t}\left\{\begin{array}{l}
i-d \\
i-d-m+1
\end{array}, y_{p}\left\{\begin{array}{l}
k \\
k-n+1
\end{array},\right.\right.\right.\right. \\
u\left\{\begin{array}{l}
k-d \\
k i-d-m+1
\end{array}: \mathbf{a}, b, K, C, \varepsilon\right) \\
i=\max (n, d+m), \cdots, N_{t}+d
\end{gathered}
$$

where $\phi$ is the nonlinear function implemented by a feed-forward SVM, $y_{p}\left\{\begin{array}{c}k \\ k-n+1\end{array}\right.$ and $u\left\{\begin{array}{l}k-d \\ k i-d-m+1\end{array}\right.$ are the known system output and input sequences respectively at time $k . \mathbf{a}=\left[a_{1}, \cdots a_{N_{t}+d-\max (n, d+m)+1}\right]^{\prime}$ and $b$ are the coefficients of SVM after learning. In order to simplify the writing, a covers all training points' coefficients including non support vectors', which values will be zero or in a very small neighborhood of zero. $K$ is the kernel function. $C$ is the penalty factor and $\varepsilon$ is the accuracy of Vapnik's loss function (see detail in [6]). Thus, provided that $n, m$ and $d$ are known, we can use $\left\{y_{p t}(i+1):\left(y_{p t}\left\{\begin{array}{l}i \\ i-n+1\end{array}, u_{t}\left\{\begin{array}{l}i-d \\ i-d-m+1\end{array}\right)\right\}\right.\right.$ to train the SVM based straight model (i.e. internal model). Also, the SVM based inverse model can be trained by $\left\{u_{t}(i-d):\left(y_{p t}\left\{\begin{array}{l}i+1 \\ i-n+1\end{array}, u_{t}\left\{\begin{array}{l}i-d-1 \\ i-d-m+1\end{array}\right)\right\}\right.\right.$ directly. But unfortunately, in most cases, systems' structure information is unknown. So in this paper, we assume that $n, m$ and $d$ are unknown.

\subsection{Straight model}


In black-box identification, how to truncate the regression series $y_{p t}$ and $u_{t}$ is important. Here we use $n^{\prime}$ and $m^{\prime}$ denote the truncation of $y_{p t}$ and $u_{t}$ respectively. Obviously, $m^{\prime}>d$ should be guaranteed. Too large $n^{\prime}$ and $m^{\prime}$ will increase the training computation, and too small $n^{\prime}$ and $m^{\prime}$ will be insufficient to make the SVM to meet the nonlinear characteristic. We can use simulation test in generalization ability to choose $n^{\prime}$ and $m^{\prime}$.

Once $n^{\prime}$ and $m^{\prime}$ are selected, the SVM based straight model will be represented as follow:

$$
\begin{gathered}
y_{m}(k+1)=\phi_{1}\left(y _ { p t } \left\{\begin{array}{l}
i \\
i-n^{\prime}+1
\end{array}, u_{t}\left\{\begin{array}{l}
i \\
i-m^{\prime}+1
\end{array}, y_{p}\left\{\begin{array}{l}
k \\
k-n^{\prime}+1
\end{array},\right.\right.\right.\right. \\
u\left\{\begin{array}{l}
k \\
k-m^{\prime}+1
\end{array}: \mathbf{a}, b, K, C, \varepsilon\right) \\
i=\max \left(n^{\prime}, m^{\prime}\right), \cdots, N_{t}
\end{gathered}
$$

where $\phi_{1}$ is another nonlinear function implemented by a feed-forward SVM. And here $\mathbf{a}=\left[a_{1}, \cdots a_{N_{t}-\max \left(n^{\prime}, m^{\prime}\right)+1}\right]^{\prime}$. Because the time delay $d$ is unknown, we use $u_{t}\left\{\begin{array}{l}i \\ i-m^{\prime}+1\end{array}\right.$ before the time instant of $i+1$ to train the straight model. As we can see, that the time delay will not increase the computation in SVM based modeling.

\subsection{Inverse model}

In order to deal with the unknown time delay, we will adopt more regression factors of $y_{p t}$ before the time instant $i$ in SVM training, which will sufficiently take the time delay into account. So the SVM based inverse model will be represented as follow:

$$
\begin{gathered}
u(k)=\phi_{2}\left(y _ { p t } \left\{\begin{array}{l}
i+l \\
i-n^{\prime \prime+1}
\end{array}, u_{t}\left\{\begin{array}{l}
i-1 \\
i-m^{\prime \prime}+1
\end{array}, r\left\{\begin{array}{l}
k+l \\
k
\end{array},\right.\right.\right.\right. \\
y_{p}\left\{\begin{array}{l}
k-1 \\
k-n^{\prime \prime+1}
\end{array}, u\left\{\begin{array}{l}
k-1 \\
k-m^{\prime \prime}+1
\end{array}: \mathbf{a}, b, K, C, \varepsilon\right)\right. \\
i=\max \left(n^{\prime \prime}, m^{\prime \prime}\right), \cdots, N_{t}
\end{gathered}
$$

where $\phi_{2}$ is another nonlinear function implemented by a feed-forward SVM. Because the future sequence of $y\left\{\begin{array}{c}k+l \\ k\end{array}\right.$ is unknown, we use he tracking set point sequence $r\left\{\begin{array}{c}k+l \\ k\end{array}\right.$ in (4) to predict the current control input. In this case, $\mathbf{a}=\left[a_{1}, \cdots a_{N_{t}-\max \left(n^{\prime \prime}, m^{\prime \prime}\right)+1}\right]^{\prime}$, $n^{\prime \prime}$ and $m^{\prime \prime}$ are the truncation number of past $y_{p t}$ and $u_{t}$ respectively. And $l \geq d+1$ must be guaranteed.

\section{SVM based control}

In this paper, the problem of tracking a sequence $\{r(k)\}$ is considered. And before we study the internal model control, the direct inverse model control strategy will be discussed first, which is widely studied in neural network field.

\subsection{Direct inverse model control}

In the case of follow-up control, we can use the direct inverse model control method, which indeed is not a close-loop control problem, though there has feedback of process output $y$ to the controller. It is like a time series predictive control strategy. The control device is an inverse model of the process only, which generate the output according to the future $l$-step $r(k)$, previous $m^{\prime \prime}$-step known input $u$ and $n^{\prime \prime}$-step known output $y$. The diagram of direct inverse model control is shown in Fig.2.

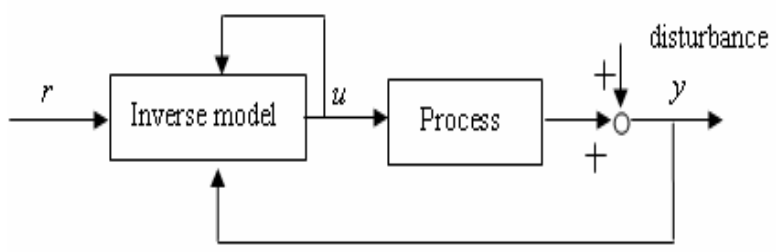

Fig.2: The diagram of direct inverse model control.

Since the controller is non adaptive, the quality of its control performance relies heavily on the fidelity of the inverse model, the system initial status and the smoothness of $r(k)$. So this control method can be only used if the inverse model is very perfect and the effect of disturbance can be completely ignored.

\subsection{Internal model control}

In order to overcome the drawbacks of direct inverse control strategy, a SVM based internal model (see Fig.1) is introduced to generate the feedback error. This error includes model mismatch and disturbance. And the controller generates corresponding output according to the set point value and error. And in this paper, we adopt a P-typed controller.

Let us denote by $P$ the nonlinear operator describing the process, by $M$ the nonlinear operator describing the SVM based internal model, by $G$ the operator describing the controller, and by Id the identity operator. And in operator representation, the system output is

$$
y=\frac{G \circ P}{I d+G \circ(P-M)} r+\frac{I d-G \circ M}{I d+G \circ(P-M)} p
$$

where $p$ denotes disturbance. The SVM based internal model will use the past information about $y$ and $u$. And we can see that if the process is stable and internal model is perfect, the stability of the control system is up to the controller.

\section{Simulation}

In order to illustrate the results of SVM based internal model control, a plant of CSTR is selected which was studied in [13]. In the CSTR a first-order irreversible exothermic reaction $A \mapsto B$ occurs. The material and energy balance equations are: 


$$
\begin{aligned}
\dot{x}_{1}(t)= & \frac{-1}{\lambda} x_{1}(t)+D_{a}\left(1-x_{1}(t)\right) \exp \left(\frac{x_{2}(t)}{1+x_{2}(t) / \gamma_{0}}\right) \\
& +\left(\frac{1}{\lambda}-1\right) x_{1}(t-\tau) \\
\dot{x}_{2}(t)= & -\left(\frac{1}{\lambda}+\beta\right) x_{2}(t)+H D_{a}\left(1-x_{1}(t)\right) \exp \left(\frac{x_{2}(t)}{1+x_{2}(t) / \gamma_{0}}\right) \\
& +\left(\frac{1}{\lambda}-1\right) x_{2}(t-\tau)+\beta u(t-\tau)
\end{aligned}
$$$$
x_{i}(t)=\theta_{i}(t) \quad \text { for } \quad \mathrm{t} \in[-\tau, 0], i=1,2 .
$$$$
x_{i}(t)=0 \quad \text { for } \quad \mathrm{t} \leq-\tau, i=1,2 \text {. }
$$

The state $x_{1}(t)$ corresponds to the conversion rate of the reaction and $0 \leq x_{1}(t) \leq 1 ; x_{2}(t)$ is the dimensionless temperature. Assume that only the temperature can be measured on line, i.e.

$$
y(t)=\left[\begin{array}{ll}
0 & 1
\end{array}\right]\left[\begin{array}{l}
x_{1}(t) \\
x_{2}(t)
\end{array}\right]
$$

The parameters are given as:

$$
\gamma_{0}=20 \quad H=8 ; \quad \beta=0.3 ; \quad D_{a}=0.072 \quad \lambda=0.8 ; \quad \tau=2 \text {. }
$$

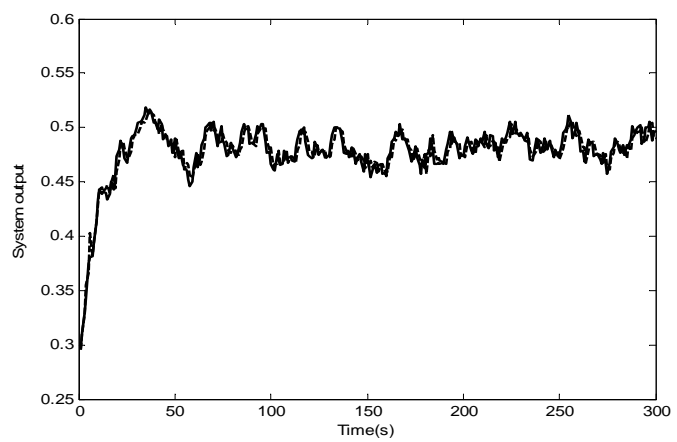

Fig.3: Straight model identification result.

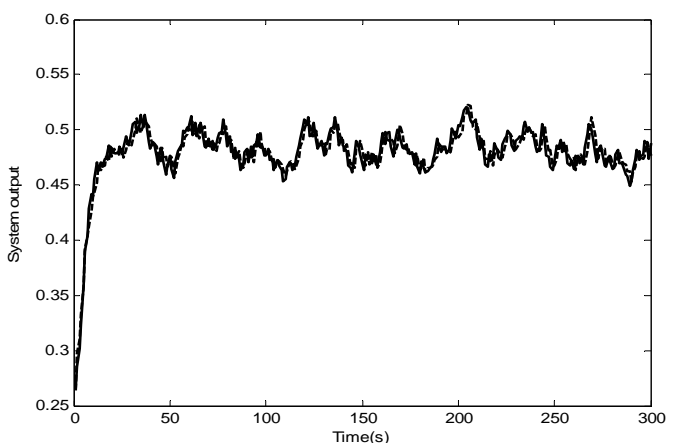

Fig.4: Straight model generalization test result 1.

In this experiment, sample time is $t_{s}=1 \mathrm{~s}$. And we select $m^{\prime}=n^{\prime}=m^{\prime \prime}=n^{\prime \prime}=l=3, C=10000, \varepsilon=0.0001$. SVM adopts quadratic polynomial kernel function $K\left(x \cdot x_{i}\right)=\left(x \cdot x_{i}+1\right)^{2}$. And we use $u \in[0,1]$ to generate one training set and two test sets.

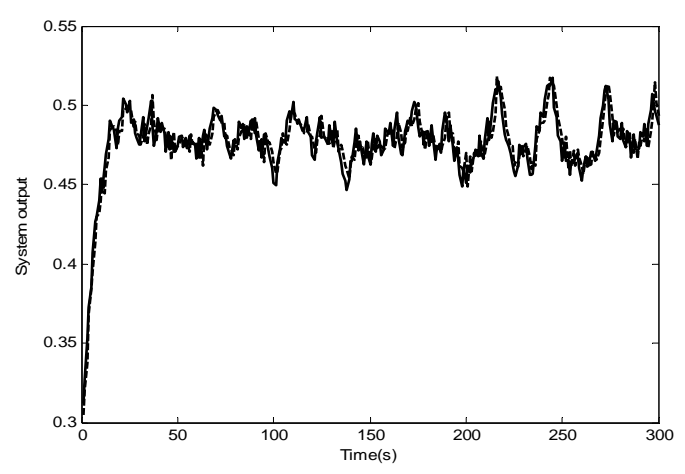

Fig.5: Straight model generalization test result 2.

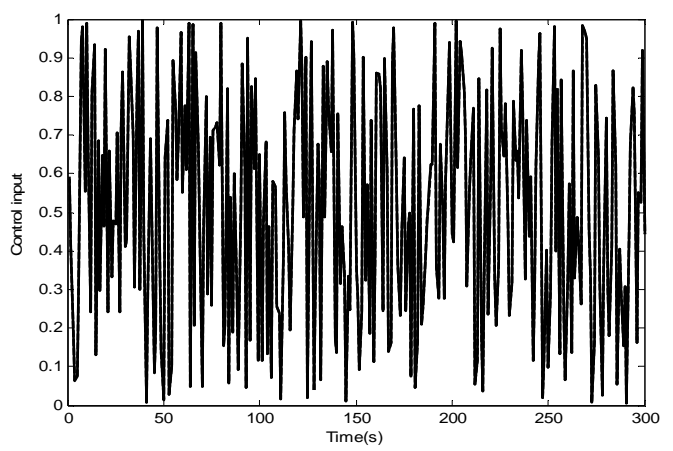

Fig.6: Inverse model identification result.

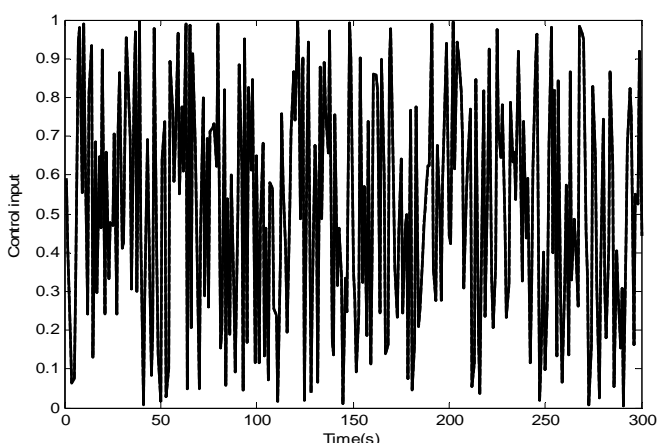

Fig.7: Inverse model generalization result 1.

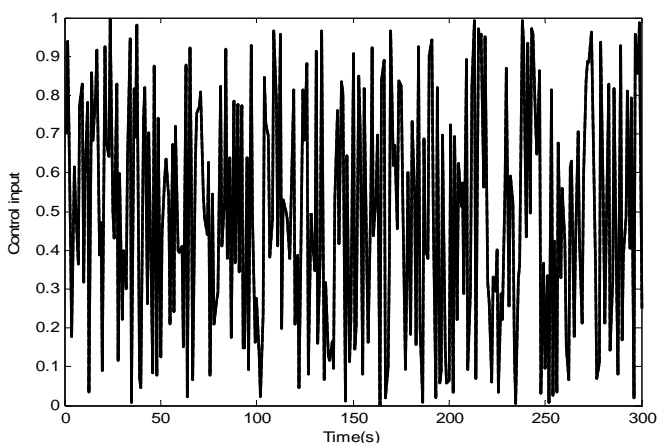

Fig.8: Inverse model generalization result 2. 
And all experiments are based on the same training set. In Fig.3 to Fig.8, solid lines denote system real output, dashed lines denote SVM identification output. Fig.3 shows the straight model identification result of the training set. Fig.4 and Fig.5 show the straight model generalization results of the other two test sets. Fig. 6 shows the inverse model identification result of the training set. Fig.7 and Fig.8 show the inverse model generalization results of the other two test sets.

From Fig.3 to Fig.8, we can see both the straight and inverse modeling results are good, and the generalization capability is also good.

In the case of direct inverse control, we discuss 2 situations. First, assume that $r(k)$ is unchangeable and $r(k)=0.6$. Fig.9 gives the control output. And we can see system can track the set point at last, but in the first 140 sample instants, it vibrates acutely because (1) inverse model always exists mismatch; (2) $r$ jumps to set point abruptly at the first sample instant; (3) we assume system is zero initial status, so regression factors $y_{p}$ and $u$ before the first sample instant are zero. Second, we choose a reference trajectory as below which smoothly leads to set point.

$$
r(k+1)=a_{r} r(k)+\left(1-a_{r}\right) r_{s p}
$$

where $r_{s p}=0.6$ is the set point value, $a_{r}=0.9$ is a tuning factor related with the control system's robustness and convergence. Fig.10 gives the simulation result. And we can see the control performance is better than that of Fig.9. System output can track the reference trajectory well and the overshot is small. And a disturbance rejection $(\mathrm{d}=0.06)$ is added when system is in steady state at time $\mathrm{k}=110 \mathrm{~s}$, and the effect on the output response is observed that after about 50 seconds the output will track the set point well again. Obviously, the re-tracking time is too long.

From Fig.9 and Fig.10, we can see the direct inverse model control strategy relies heavily on the fidelity of the inverse model, the system initial status and the smoothness of $r(k)$. And the performance in dealing with disturbance rejection is not satisfying. So in the following, we will illustrate the simulation results of SVM based internal model control. In this control strategy, the internal model adopts SVM based straight model and the controller adopts the P-typed controller.

Fig.11 shows the simulation results when $K_{p}=1.5, K_{p}=2$ and $K_{p}=2.5$. And we find that system outputs are smooth. And when $K_{p}=2$, the control performance is very good. Now, let's see Fig.3, Fig.4 and Fig.5, and we can easily see that the nonlinear system's stable gain is about 0.5. This is why the control performance is good when $K_{p}=2$, which is the inverse of the nonlinear system's stable gain.

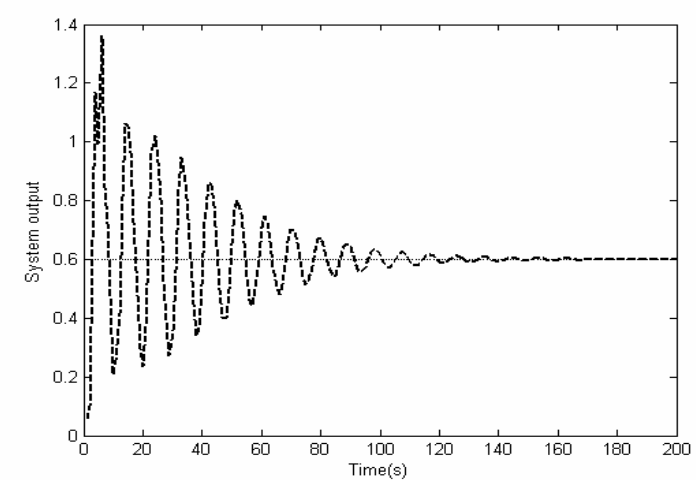

Fig.9: Direct inverse model control output 1.

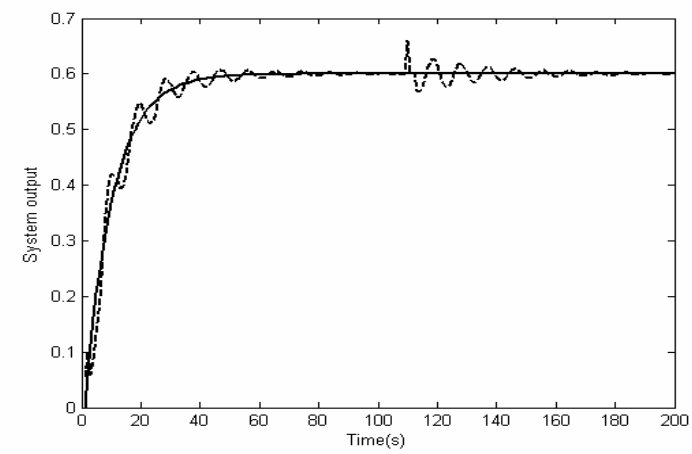

Fig.10: Direct inverse model control output 2.

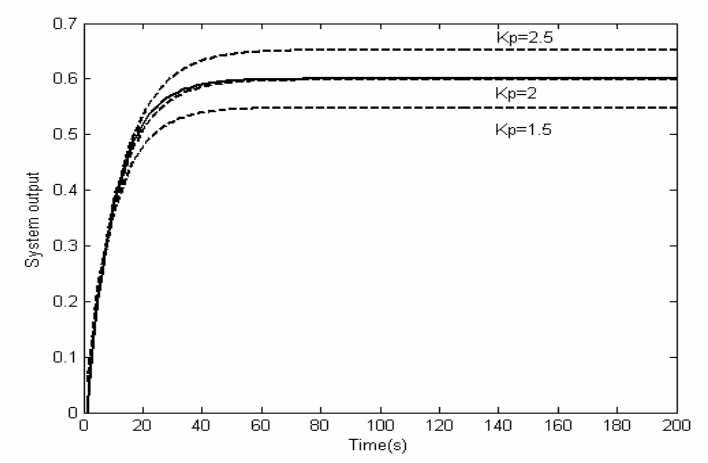

Fig.11:Internal model control outputs when $K_{p}=1.5, K_{p}=2$ and $K_{p}=2.5$.

Finally, the system's performance is investigated under the influence of additive noise (random number between $2 \%$ of $r_{s p}$ ) and a disturbance rejection (d=0.06) when $K_{p}=2$. Fig.12 shows the simulation results. And the performance in dealing with noise and disturbance rejection is very good.

\section{Conclusion}

In this paper, the design procedure of SV based inverse and straight model identification methods for stable nonlinear discrete-time process with input- 
output form and time delay are discussed in detail. And direct inverse control and internal model control strategies for such system are proposed based on the SVM models. The control of a simulated CSTR process illustrates that the proposed design procedures are feasible and the properties of the SVM based model identification are good. Also, it shows that the direct inverse model control strategy relies heavily on the fidelity of the inverse model, the system initial status and the smoothness of the reference trajectory of set point value. Further more, it shows that the performance of internal model control strategy is better than that of direct inverse control in stabilization and robustness.

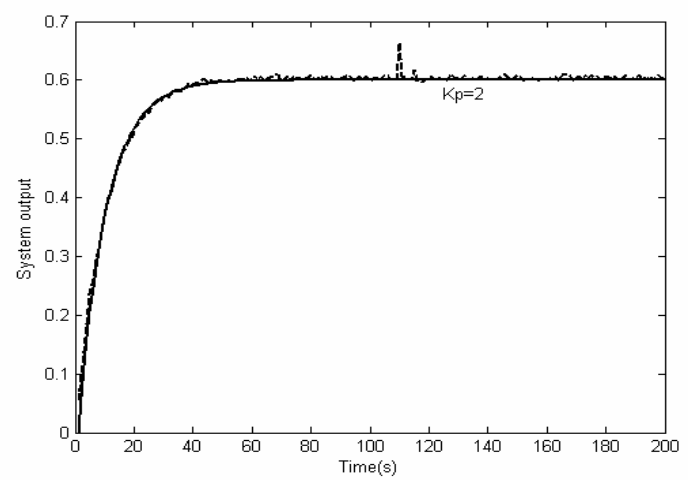

Fig.12: Internal model control output when $K_{p}=2$ and disturbance rejection and noise exist.

\section{Acknowledgement}

This work is partially supported by National Science Fund for Distinguished Young Scholars (Grant No. 60625302), Major State Basic Research Development Program of China (973 Program, Grant No. 2002CB312200), National High-Tech Research and Development Program of China (863 Program, Grant No. 20060104Z1081) and Major State Basic Research Development Program of Shanghai (Grant No. 05DJ14002).

\section{References}

[1] R. Boukezzoula, S. Galichet and L. Foulloy, Nonlinear internal model control: application of inverse model based fuzzy control. IEEE Trans. On fuzzy systems, 11: 814-829, 2003.

[2] I. Rivals and L. Personnaz, Nonlinear internal model control using neural networks: application to processes with delay and design issues. IEEE Trans. On Neural Networks, 11: 80-90, 2000.
[3] J. Calvet and Y. Arkun, Feed forward and feedback linearization of nonlinear systems and its implementation using internal model control. Ind.Eng.Chem.Res, 27: 1822-1831, 1988.

[4] E.C. Morles and N. Mort, Inverse model neural network-based control of dynamic system. Prof. of International conference on control 1994, 2:955960, 1994.

[5] J. Alvarez, An internal-model controller for nonlinear systems. Prof. of the $3^{\text {rd }}$ European Control Conference, pp.301-306, 1995.

[6] V.N. Vapnik, The nature of statistical learning theory, Springer-Verlag, 1995.

[7] C. Cortes, Prediction of generalization ability in learning machines, Ph. D. Thesis, Rochester, Univ. New York, USA, 1995.

[8] A.J. Smola and B. Schölkopf, A tutorial on support vector regression, Neurocolt technical report, Royal Holloway College, Unisversity of London, 1998.

[9] P.M.L. Drezet and R.F. Harrison, Support vector machines for system identification. Prof. of the International Conference on CONTROL'98, pp.668-692, 1998.

[10] J.L.R. Alvarez, Support vector method for robust ARMA system identification. IEEE Trans. on Signal Processing, 52(1):155-164,2004

[11] D.C.Wang and T.J.Fang, Internal model control approach based on support vector machines. Control Theory \& Application, 21: 85-88, 2004 (in Chinese).

[12] W.M.Zhong, D.Y.Pi and Y.X.Sun, SVM based direct inverse-model identification. Control Theory \& Applications, 22:307-310, 2005 (in Chinese).

[13] Y.Y. Cao and P.M. Frank, Analysis and synthesis of nonlinear time-delay systems via fuzzy control approach. IEEE transactions on Fuzzy Systems, 8: 200-211, 2000. 